\title{
Long non-coding RNA MEG3 inhibits NSCLC cells proliferation and induces apoptosis by affecting p53 expression
}

Kai-hua Lu, ${ }^{1,4 \dagger}$, Wei Li ${ }^{1 \dagger}$, Xiang-hua Liư ${ }^{2 \dagger}$, Ming Sun ${ }^{2}$, Mei-ling Zhang ${ }^{1}$, Wei-qin Wu' ${ }^{1}$, Wei-ping Xie ${ }^{3 *}$ and Ya-yi Hou ${ }^{4^{*}}$

\begin{abstract}
Background: Long non-coding RNAs play an important role in tumorigenesis, hence, identification of cancerassociated IncRNAs and investigation of their biological functions and molecular mechanisms are important for understanding the development and progression of cancer. Recently, the downregulation of IncRNA MEG3 has been observed in various human cancers. However, its role in non-small cell lung cancer (NSCLC) is unknown. The aim of this study was to examine the expression pattern of MEG3 in NSCLC and to evaluate its biological role and clinical significance in tumor progression.
\end{abstract}

Methods: Expression of MEG3 was analyzed in 44 NSCLC tissues and 7 NSCLC cell lines by qRT-PCR. Overexpression approaches were used to investigate the biological functions of MEG3 in NSCLC cells. Bisulfite sequencing was used to investigate DNA methylation on MEG3 expression. The effect of MEG3 on proliferation was evaluated by MTT and colony formation assays, and cell apoptosis was evaluated by Hoechst staining and Flow-cytometric analysis. NSCLC cells transfected with PCDNA-MEG3 were injection into nude mice to study the effect of MEG3 on tumorigenesis in vivo. Protein levels of MEG3 targets were determined by western blot analysis. Differences between groups were tested for significance using Student's $t$-test (two-tailed).

Results: MEG3 expression was decreased in non-small cell lung cancer (NSCLC) tumor tissues compared with normal tissues, and associated with advanced pathologic stage, and tumor size. Moreover, patients with lower levels of MEG3 expression had a relatively poor prognosis. Overexpression of MEG3 decreased NSCLC cells proliferation and induced apoptosis in vitro and impeded tumorigenesis in vivo. MDM2 and p53 protein levels were affected by MEG3 over-expression in vitro.

Conclusions: Our findings indicate that MEG3 is significantly down-regulated in NSCLC tissues that could be affected by DNA methylation, and regulates NSCLC cell proliferation and apoptosis, partially via the activition of p53. Thus, MEG3 may represent a new marker of poor prognosis and is a potential therapeutic target for NSCLC intervention.

Keywords: Long non-coding RNA, MEG3, NSCLC, Proliferation, p53

\footnotetext{
*Correspondence: wpxie@njmu.edu.cn; yayihou@nju.edu.cn

${ }^{\dagger}$ Equal contributors

${ }^{3}$ Department of respiratory, First Affiliated Hospital, Nanjing Medical University, Nanjing, People's Republic of China

${ }^{4}$ Immunology and Reproductive Biology Lab of Medical School and State Key Laboratory of Pharmaceutical Biotechnology, Nanjing University, Nanjing,

People's Republic of China

Full list of author information is available at the end of the article
}

\section{Biomed Central}

(c) 2013 Lu et al.; licensee BioMed Central Ltd. This is an open access article distributed under the terms of the Creative Commons Attribution License (http://creativecommons.org/licenses/by/2.0), which permits unrestricted use, distribution, and reproduction in any medium, provided the original work is properly cited. 


\section{Background}

Non-small cell lung cancer (NSCLC) including adenocarcinoma and squamous cell carcinoma, is a predominant form of lung cancer, and accounts for the majority of lung cancer associated deaths worldwide [1]. Despite the recent advances in clinical and experimental oncology, the prognosis of lung cancer is still unfavorable, with a 5-year overall survival rate of approximately $11 \%$ [2]. Thus, a detailed understanding of the mechanisms underlying NSCLC development and progression is essential for improving diagnosis, prevention and treatment of this disease. Recently, there is growing evidence indicating that non-coding RNAs may be involved in NSCLC pathogenesis, providing new insights into the biology of this disease [3,4].

Recent improvements in high-throughput transcriptome analysis in the last few years, have led to the discovery that $>90 \%$ of the total mammalian genome can be transcribed and may yield many short or long noncoding RNAs (lncRNAs) with limited or no protein-coding capacity $[5,6]$. Although many studies have helped unraveling the function of microRNAs, the lncRNAs counterpart of the transcriptome is less well characterized. lncRNAs are known to play important roles during cellular development and differentiation, and a large range of functions, such as modulation of proliferation and invasiveness of tumors [7], and reprogramming of induced pluripotent stem cells [8] have been attributed to IncRNAs. Dysregulation of some lncRNAs has been shown in various types of cancers, such as breast cancer, hepatocellular carcinoma, melanoma, bladder cancer, and prostate cancer [7,9-14]. One such lncRNA, HOTAIR, has been determined as a negative prognostic indicator in breast, liver and pancreatic cancer patient survival, evidencing a close association with breast cancer cell metastasis $[7,15,16]$. Recent studies have also revealed the contribution of lncRNAs, as proto-oncogenes (e.g. ANRIL) and tumor suppressor genes (e.g. MEG3) in tumorigenesis $[17,18]$.

Maternally expressed gene 3 (MEG3), an lncRNA, is expressed in many normal tissues. However, MEG3 expression is lost in an expanding list of primary human tumors, and promoter hypermethylation or hypermethylation of the intergenic differentially methylated region has been shown to contribute to the loss of MEG3 expression in tumors $[19,20]$. MEG3 represents as a tumor suppressor gene, and its ectopic expression can inhibit cell proliferation and promote cell apoptosis in human glioma cell lines [21]. Moreover, accumulation of p53 (TP53) protein and its target gene expression partly contribute to cell growth inhibition induced by MEG3 [22]. However, very little is known about MEG3 expression level in NSCLC, and its role in NSCLC development.

In this study, we demonstrated that $M E G 3$ expression was significantly decreased in NSCLC tissues compared to adjacent normal tissues. The correlation between MEG3 downregulation and advanced pathologic stage, tumor size, and patient survival time was also explored. Moreover, ectopic expression of MEG3 inhibited cell proliferation and promoted cell apoptosis in human NSCLC cell lines and overexpression of MEG3 was able to impede the development of tumors in vivo. We further verified that overexpression of MEG3 could induce the activation of p53. Taken together, this study indicated that lncRNA, especially MEG3 plays an important role in NSCLC development and could be a potential therapeutic target for patients with NSCLC.

\section{Methods}

\section{Patient and tissue samples}

Paired NSCLC and adjacent non-tumor lung tissues were obtained from 44 patients who underwent primary surgical resection of NSCLC between 2006 and 2007 at First Affiliated Hospital of Nanjing Medical University, China. NSCLC and normal tissues were immediately snap-frozen in liquid nitrogen and stored at $-80^{\circ} \mathrm{C}$ until total RNA was extracted. Tumor samples were at least $80 \%$ composed of viable-appearing tumor cells on histological assessment. The pathological stage, grade and nodal status were appraised by an experienced pathologist. Clinicopathologic characteristics including tumornode-metastasis (TNM) staging were also collected. The study was approved by the Research Ethics Committee of Nanjing Medical University, China. Informed written consents were obtained from all patients who participated in this study.

\section{Cell lines and culture conditions}

Six NSCLC adenocarcinoma cell lines (A549, SPC-A1, NCI-H1650， NCI-H358， NCI-H1299， NCI-H1975), a NSCLC squamous carcinomas cell line (SK-MES-1), and a normal human bronchial epithelial cell line (16HBE) were purchased from the Institute of Biochemistry and Cell Biology of the Chinese Academy of Sciences (Shanghai, China). 16HBE, A549, NCI-H1650, NCI-H358, NCI-H1975 and NCI-H1299 cells were cultured in RPMI 1640 medium; SPC-A1, and SK-MES-1 cells were cultured in DMEM (GIBCO-BRL) medium, supplemented with $10 \%$ fetal bovine serum $(10 \% \mathrm{FBS})$, $100 \mathrm{U} / \mathrm{ml}$ penicillin, and $100 \mathrm{mg} / \mathrm{ml}$ streptomycin (Invitrogen, Shanghai, China) in humidified air at $37^{\circ} \mathrm{C}$ with $5 \% \mathrm{CO}_{2}$.

\section{RNA extraction and qRT-PCR analysis}

Total RNA was isolated with TRIzol reagent (Invitrogen, Carlsbad, CA, USA) according to the manufacturer's protocol. 500 ng total RNA was reverse transcribed in a final volume of $10 \mu \mathrm{l}$ using random primers under standard conditions using the PrimeScript RT reagent Kit. 
Assays were performed to detect MEG3 expression using the PrimeScript RT reagent Kit and SYBR Premix Ex Taq (TaKaRa, Dalian, China) according to the manufacturer's instructions.

The relative levels of $M E G 3$ were determined by qPCR using gene specific primers. GAPDH was measured as an internal control, as its expression showed minimal variation in different cell lines and cancer specimens. The RT reaction was carried out under the following conditions: $37^{\circ} \mathrm{C}$ for $15 \mathrm{~min} ; 85^{\circ} \mathrm{C}$ for $5 \mathrm{sec}$; and then held on $4^{\circ} \mathrm{C}$. After the RT reaction, 1 ul of the complementary DNA was used for subsequent qRT-PCR reactions. The PCR primers for MEG3 or GAPDH were as follows: MEG3 sense, 5' CTGCCCATCTACACCTCACG 3' and reverse, 5' CTCTCCGCCGTCTGCGCTAGGGGCT 3'; GAPDH sense, 5' GTCAACGGATTTGGTCT GTATT 3' and reverse, 5' AGTCTTCTGGGTGGCAGTGAT 3'. The PCR reaction was conducted at $95^{\circ} \mathrm{C}$ for $30 \mathrm{~s}$ and followed by 40 cycles of $95^{\circ} \mathrm{C}$ for $5 \mathrm{~s}$ and $60^{\circ} \mathrm{C}$ for $34 \mathrm{~s}$ in the ABI 7500 real-time PCR system (Applied Biosystems, Foster City, CA, USA). The qPCR results were analyzed and expressed as relative mRNA expression of CT (threshold cycle) value, which was then converted to fold changes.

\section{Methylation analysis of CpG island}

For determination of methylation status of the CpG island, genomic DNA prepared from NSCLC cells and normal tissues, was modified by sodium bisulfite (EZ DNA Methylation Kit , Zymo Research), followed by PCR using the sense primer $5^{\prime}$ TTTTTTTGTTGTAATTTGGGTG 3' and reverse, 5' ACGAATACCGTCTTCCTTTTAC 3', respectively. PCR-amplified product was transformed in $\mathrm{E}$. coli DH5 $\alpha$ cells. Subsequently obtained plasmids were subjected to sequencing.

\section{Treatment of SPC-A1 cells with 5-aza-2-deoxy-cytidine (5-aza-CdR)}

SPC-A1 cells $\left(2.5 \times 10^{5}\right)$ were seeded into six-well culture plate on day 0 and exposed to 0,2 or $5 \mu \mathrm{M} 5$-aza-CdR (Sigma-Aldrich, USA)from day 1 to day 3 . The cells treated with 5 -aza-CdR were harvested on day 3 and used for detection of MEG3 expression.

\section{Plasmid constructs}

The sequence of MEG3 was synthesized and subcloned into pCDNA3.1 (Invitrogen, Shanghai, China). Ectopic expression of MEG3 was achieved by using the pCDNAMEG3 transfection and empty pCDNA vector (empty) was used as control. The expression level of MEG3 was detected by qPCR.

\section{Transfection of NCSCL cells}

All plasmid vectors (pCDNA-MEG3 and empty vector) for transfection were extracted by DNA Midiprep or
Midiprep kit (Qiagen, Hilden, Germany). SPC-A1 and A549 cells cultured on six-well plate were transfected with the pCDNA -MEG3 or empty vector using Lipofectamine2000 (Invitrogen, Shanghai, China) according to the manufacturer's instructions. Cells were harvested after 48 hours for qRT-PCR and western blot analyses.

\section{Cell proliferation assays}

Cell proliferation was monitored using Cell Proliferation Reagent Kit I (MTT) (Roche Applied Science). pCDNAMEG3 and empty vector transfected SPC-A1 cells (3000/ well) were allowed to grow in 96-well plates. Cell proliferation was measured every 24 hours following the manufacturer's protocol. All experiments were performed in quadruplicate. For colony formation assay, a total of 500 pCDNA-MEG3 and empty vector cells were placed in a fresh six-well plate and maintained in media containing $10 \%$ FBS, replacing the medium every 4 days. After 14 days, cells were fixed with methanol and stained with $0.1 \%$ crystal violet (Sigma-Aldrich (country???)). Visible colonies were manually counted. Triplicate wells were measured for each treatment group.

\section{Flow-cytometric analysis of apoptosis}

SPC-A1 and A549cells transfected with pCDNA-MEG3 and empty vector were harvested 48 hours after transfection by trypsinization. Following double staining with FITC-Annexin V and Propidium iodide (PI), the cells were analyzed using flow cytometry (FACScan ${ }^{\oplus}$; BD Biosciences) equipped with a CellQuest software (BD Biosciences) [23]. Cells were discriminated into viable cells, dead cells, early apoptotic cells, and apoptotic cells. The percentage of early apoptotic cells were compared to control groups from each experiment. All of the samples assayed were in triplicates.

\section{Hoechst staining assay}

SPC-A1 and A549 cells transfected with pCDNA-MEG3 and empty vector were cultured in six-well plates, and were incubated with Hoechst 33342 solution $(50 \mathrm{ng} / \mathrm{ml}$, Sigma-Aldrich, St Louis, MO, USA) for $10 \mathrm{~min}$ at room temperature. Cells were then washed twice with PBS and changes in nuclear morphology were detected by fluorescence microscopy using $365 \mathrm{~nm}$ filter for Hoechst 33342. For quantification of Hoechst 33342 staining, the percentage of Hoechst -positive nuclei per optical field (at least 50 fields) was counted in three independent experiments.

\section{Tumor formation assay in a nude mouse model}

Female athymic BALB/c nude mice aged 4 weeks were maintained under specific pathogen-free conditions and manipulated according to protocols approved by the Shanghai Medical Experimental Animal Care Commission. SPC-A1 cells were transfected with pCDNA-MEG3 
and empty vector and harvested from six-well cell culture plates, washed with PBS, and resuspended at a concentration of $2 \times 10^{7}$ cells $/ \mathrm{mL}$. A volume of $0.1 \mathrm{~mL}$ of suspended cells was subcutaneously injected into a single side of the posterior flank of each mouse. Tumor growth was examined every three days, and tumor volumes were calculated using the equation $\mathrm{V}=0.5 \times$ $\mathrm{D} \times \mathrm{d}^{2}(\mathrm{~V}$, volume; $\mathrm{D}$, longitudinal diameter; $\mathrm{d}$, latitudinal diameter) [16]. At 3 weeks post injection, mice were euthanized, and the subcutaneous growth of each tumor was examined.

This study was carried out in strict accordance with the recommendations in the Guide for the Care and Use of Laboratory Animals of the National Institutes of Health. The protocol was approved by the Committee on the Ethics of Animal Experiments of the Nanjing medical University (Permit Number: 200933). All surgery was performed under sodium pentobarbital anesthesia, and all efforts were made to minimize suffering in mice [24].

\section{Western blotting assay}

Cells were lysed using mammalian protein extraction reagent RIPA (Beyotime china) supplemented with protease inhibitors cocktail (Roche. Switzerland) and PMSF (Roche, Switzerland). Protein concentration was measured with the Bio-Rad protein assay kit. $50 \mu \mathrm{g}$ protein extractions were separated by $12 \%$ SDS-polyacrylamide gel electrophoresis (SDS-PAGE), then transferred to $0.22 \mu \mathrm{m}$ nitrocellulose membranes (Sigma-Aldrich. USA)and incubated with specific antibodies. ECL chromogenic substrate was used to visualize the bands and the intensity of the bands was quantified by densitometry (Quantity One software; Bio-Rad). GAPDH was used as control. GAPDH antibody was purchased from sigma-Aldrich (USA), P53 antibody was purchased from Santa Cruz Biotechnology (Santa Cruz, CA, USA), P21 antibody was purchased from Cell Signaling Technology (MA, USA).

\section{Statistical Analysis}

Student's $t$-test (two-tailed), One-way ANOVA and Mann-Whitney test were performed to analyze the data using SPSS 16.0 software. $P$ values less than 0.05 were considered statistically significant.

\section{Results}

\section{MEG3 expression is downregulated in human NSCLC} tissues

qRT-PCR analysis was used to measure MEG3 expression in 44 NSCLC tissues and normal counterparts. The expression of MEG3 was significantly downregulated in NSCLC tissues (Figure 1A). Furthermore, correlation analysis of MEG3 expression with clinical pathological features of NSCLC patients, revealed a significant association between MEG3 downregulation and advanced pathological stage (I/
II,37; IIIa/b,IV,7) and NSCLC tumor size (Figure 1B,C). However, MEG3 expression was not correlated with histological subtype, patient age, gender, or tumor position (Figure 1D and Table 1). Clinical data of individual patients is shown in Additional file 1: Table S1.

Kaplan-Meier survival analysis and log-rank tests using patient postoperative survival were performed to further evaluate the correlation between MEG3 expression and NSCLC patient prognosis. According to the median ratio of relative MEG3 expression (0.27) in tumor tissues, the 44 NSCLC patients were classified into two groups: High-MEG3 group ( $n=21, M E G 3$ expression ratio $\geq$ mean ratio) and Low-MEG3 group ( $n=21$, MEG3 expression ratio $\leq$ mean ratio). The Kaplan-Meier survival curve showed that patients with decreased MEG3 expression levels had significantly shorter survival times than those with high MEG3 expression levels (Figure 1D). These findings support the hypothesis that decreased $M E G 3$ expression plays a key role in NSCLC development and progression.

\section{Effect of DNA methylation on MEG3 expression}

We next performed qRT-PCR analysis to examine the expression of MEG3 in 7 human NSCLC cell lines, including both adenocarcinoma and squamous carcinoma subtypes. Of these, five cell lines (A549, SPC-A1, NCI-H1650, NCI-H1975 and SK-MES-1) expressed lower levels of MEG3 compared with the normal bronchial epithelial cell line and 16HBE, while NCI-H358 and $\mathrm{H} 1299$ cells expressed relatively higher endogenous levels of MEG3 (Figure 2A). The expression of lncRNA is more cell sepecific [25], which may contribute to the different expression level of MEG3 in NSCLC cell lines.

The expression of $M E G 3$ was frequently downregulated in NSCLC, and hypermethylation of MEG3-MDR has been reported to be involved in MEG3 transcriptional inactivation. Following treatment of SPC-A1 cells with DNA demethylating agent (5-aza-CdR), we found that MEG3 expression was significantly increased by 1.95 - or 3.08 fold in 5-aza-CdR treated cells compared with control (Figure 2B). Moreover, among the three canonical CpG island of MEG3-DMR loci (DMR1, DMR2 and DMR3), we examined the methylation pattern of DMR2 in NSCLC and normal tissues by bisulfite sequencing, and the average frequency of methylation was $68 \%$ in normal tissues and $96 \%$ in NSCLC tissues (Figure 2C). These results indicate that downregulation of MEG3 observed in NSCLC cells might have been partly due to hypermethylation of MEG3-DMR.

\section{Effect of MEG3 on cell proliferation in vitro}

MEG3 was overexpressed in SPC-A1 and A549 cells by transfecting them with pCDNA-MEG3. qRT-PCR analysis of $M E G 3$ levels revealed that MEG3 expression was 


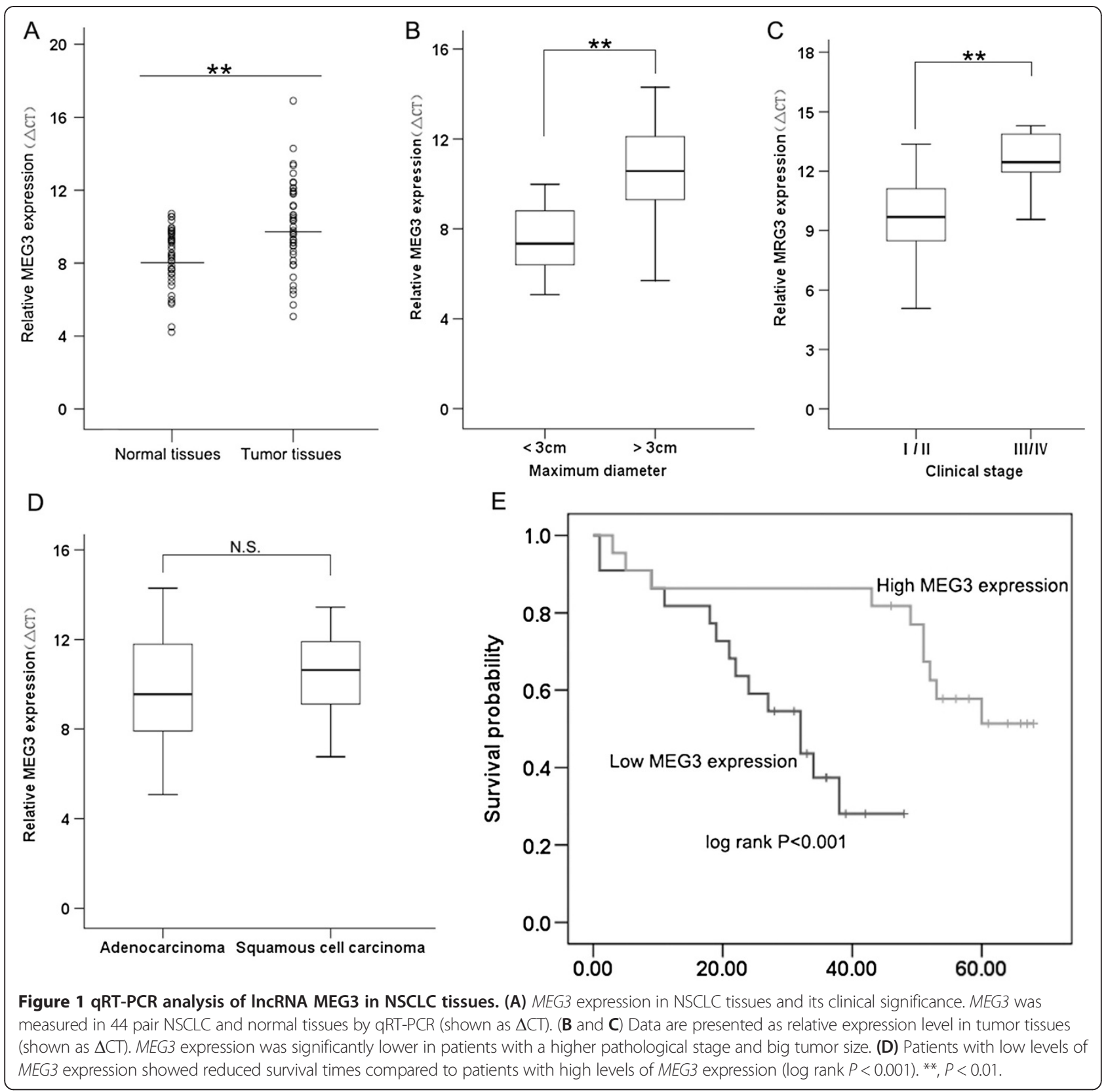

increased by 80 -fold or 91 -fold in SPC-A1 or A549 cells respectively following transfection with pCDNA-MEG3 compared with control (Figure 3A).

To assess the biological role of MEG3 in NSCLC, we investigated the effects of targeted overexpression of MEG3 on cell proliferation. MTT assay revealed that cell growth was significantly impaired in SPC-A1 and A549 cells transfected with pCDNA-MEG3 compared with controls (Figure 3B). Similarly, the results of colonyformation assays revealed that clonogenic survival was decreased following enhanced MEG3 expression in SPCA1 and A549 cells (Figure 3C). To further examine whether the effect of MEG3 on proliferation of NSCLC cells was on cell cycle regulation, cell cycle progression was analyzed by flow cytometry. The results revealed that SPC-A1 and A549 cells transfected with pCDNAMEG3 had an obvious cell cycle arrest at the G1/G0 phase and had a decreased G2/S phase (Figure 3D). Moreover, inhibition of MEG3 expression in H1299 cells promoted cells proliferation (Additional file 2: Figure S1)

\section{Effect of MEG3 on cell apoptosis and invasion}

To determine whether apoptosis was a contributing factor to cell growth inhibition, we performed Hochest 
Table 1 Correlation of the expression of MEG3 with clinicopathologic features

\begin{tabular}{llll}
\hline $\begin{array}{l}\text { Clinicopathologic } \\
\text { features }\end{array}$ & $\mathbf{n}(\%)$ & $\begin{array}{l}\text { Relative expression } \\
\text { of } \mathbf{M E G 3}^{\mathbf{a}}\end{array}$ & P-value $^{\mathbf{b}}$ \\
\hline Gender & $34(77)$ & 0.36 & $\mathrm{P}=0.653$ \\
Male & $10(23)$ & 0.42 & $\mathrm{P}=0.758$ \\
$\begin{array}{l}\text { Female } \\
\text { Site of tumor }\end{array}$ & $19(43)$ & 0.31 & \\
$\begin{array}{l}\text { Left lung } \\
\text { Right lung }\end{array}$ & $25(57)$ & 0.39 & $\mathrm{P}=0.073$ \\
Differentiation & $16(36)$ & 0.27 & \\
Poor & $28(64)$ & 0.45 & $\mathrm{P}=0.042$ \\
Moderate & & & \\
Lymph node metastasis & $27(61)$ & 0.42 & \\
Yes & $17(39)$ & 0.64 & \\
No & & & \\
\hline
\end{tabular}

Correlation of the expression of MEG3 with clinicopathologic features. a Median of relative expression. $\mathrm{b} P<0.05$ was considered significant (Mann-Whitney $U$ test between 2 groups and Kruskall-Wallis test for 3 groups). staining and flow-cytometric analysis after transfection with pCDNA-MEG3. The apoptotic rate of SPC-A1 and A549 cells transfected with pCDNA-MEG3 increased by approximately $11 \%$ and $12 \%$ respectively in comparison with cells transfected with empty vector (Figure 4A,B).

Cell invasion is a significant aspect of cancer progression, and involves the migration of tumor cells into contiguous tissues and the dissolution of extracellular matrix proteins. To investigate whether MEG3 had a direct functional role in facilitating cell invasion in NSCLC, we evaluated cancer cell invasion through transwell matrigel assay. However, alteration of MEG3 expression had no significant effects on cell invasion compared with control (data not shown).

\section{MEG3 inhibits tumorigenesis of NSCLC cells in vivo}

To explore whether the level of MEG3 expression affects tumorigenesis, pCDNA-MEG3 and empty vector stably-transfected SPC-A1 cells were inoculated into female nude mice. Eighteen days after injection, the

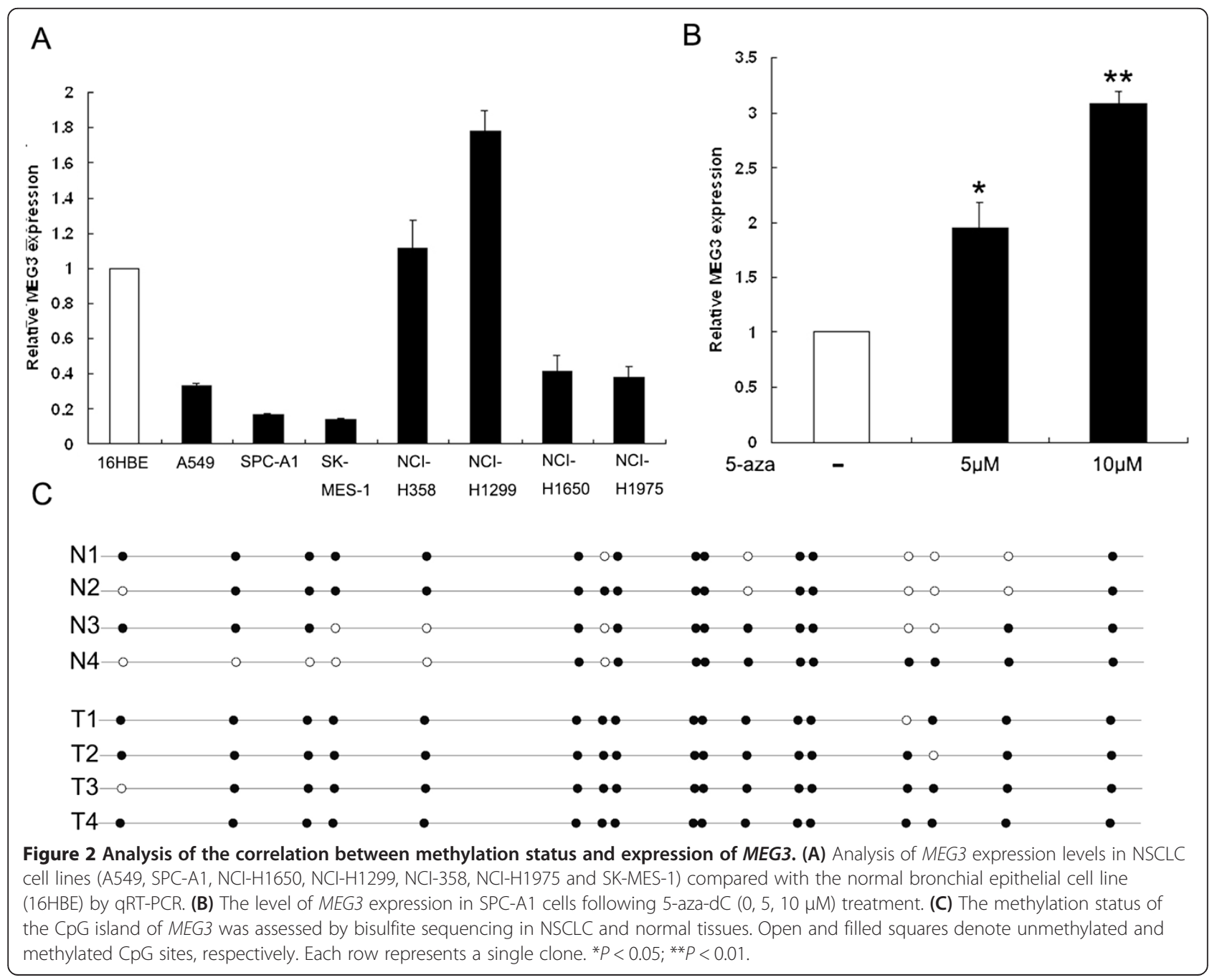




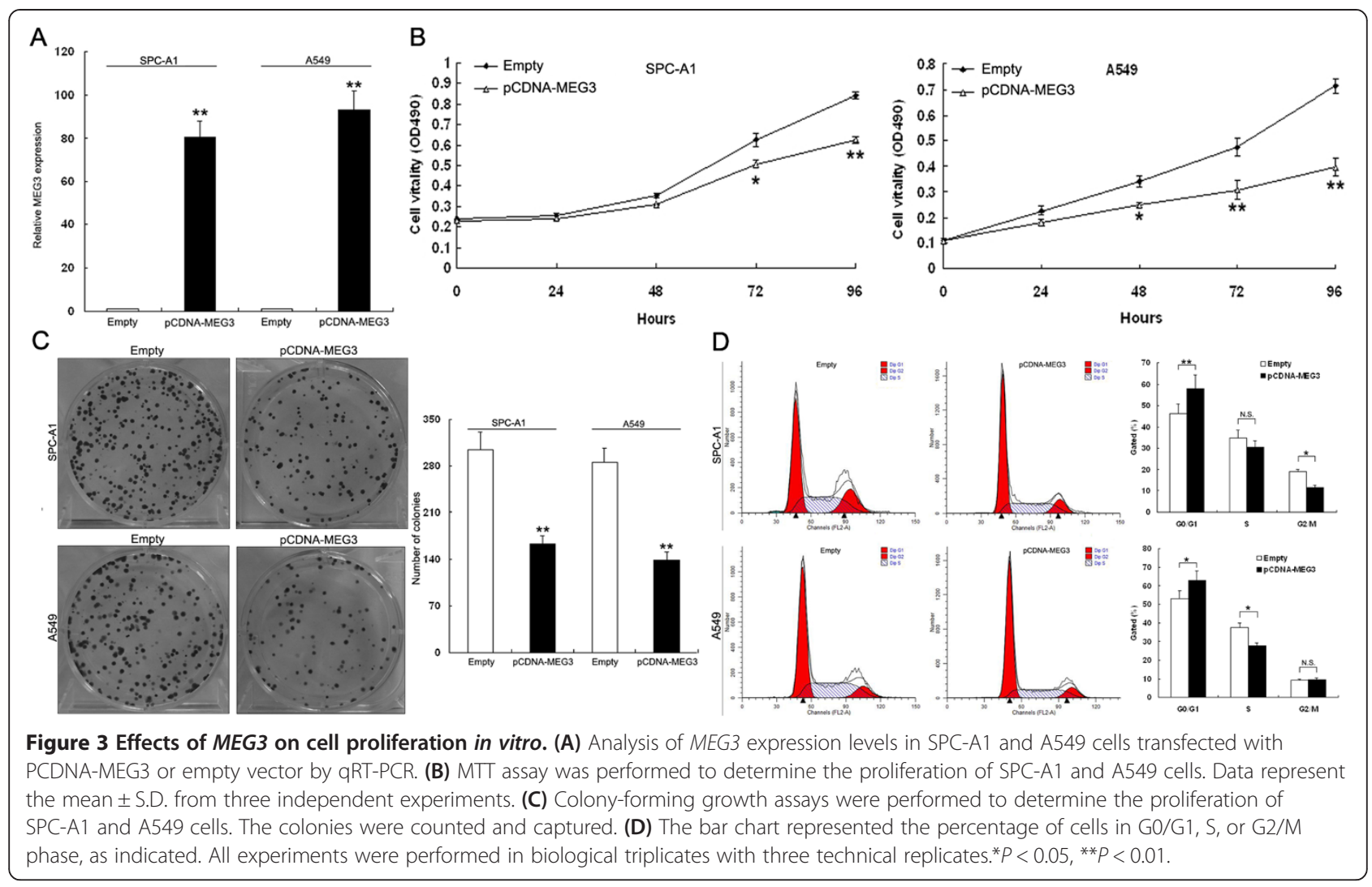

tumors formed in pCDNA-MEG3 group were substantially smaller than those in the empty vector group (Figure 5A). Moreover, the mean tumor weight at the end of the experiment was markedly lower in the pCDNAMEG3 group $(0.35 \pm 0.11 \mathrm{~g})$ compared to the control group (0.81 $\pm 0.15 \mathrm{~g})$ (Figure $5 \mathrm{~B})$. qRT-PCR analysis of $M E G 3$ expression was then performed in selected tumor tissues. The results showed that the levels of MEG3 expression in tumor tissues formed from pCDNA-MEG3 cells were higher than those of tumors formed in control group (Figure 5C). Immunostaining was used to analyze PCNA protein expression in resected tumor tissues. PCNA levels in tumors formed from control cells (empty vector), exhibited decreased positivity for PCNA than in tumors from pCDNA-MEG3 transfected SPC-A1 cells (Figure 5D). These results indicate that overexpression of MEG3 could inhibit tumor growth in vivo.

\section{MEG3 stimulates activation of p53 protein}

Further exploration of the mechanisms involved in MEG3 overexpression induced growth arrest and apoptosis was done by examining the expression of p53 protein after transfection with pCDNA-MEG3 or empty vector. Recent studies have indicated that lncRNAs may play an important role in the regulation of cell growth by modulating p53 pathway [26]. The results of western blot analysis showed that the expression of p53 was significantly increased and the expression of MDM2 was downregulated in SPC-A1 cells transfected with pCDNA-MEG3 compared to those with empty vector. No significant differences were observed in the expression levels of p21 in SPC-A1 cells transfected with pCDNA-MEG3 compared to those with empty vector (Figure 6). These data confirm that MEG3 functions as a tumor suppressor gene by regulating p53 activation in NSCLC.

\section{Discussion}

Recently, genome-wide surveys have revealed that the human genome contains $\sim 20000$ protein-coding genes and $>98 \%$ of the total genome can be transcribed, yielding many short or long noncoding RNAs (lncRNAs) with limited or no protein-coding capacity $[27,28]$. There are over 3000 human lncRNAs greater than 200nt in length, but less than $1 \%$ of them have been characterized $[5,29]$. Although only a minority have been characterized in detail, recent studies showed that lncRNAs participates in diverse biological processes including cell cycle control and cell differentiation through distinct mechanisms, such as imprinting, chromosome dosage-compensation, epigenetic regulation, mRNA splicing, nuclear and cytoplasmic trafficking [30-32]. Several studies have further demonstrated that lncRNAs are efficiently regulated during development in response to diverse signaling, and dysregulation 

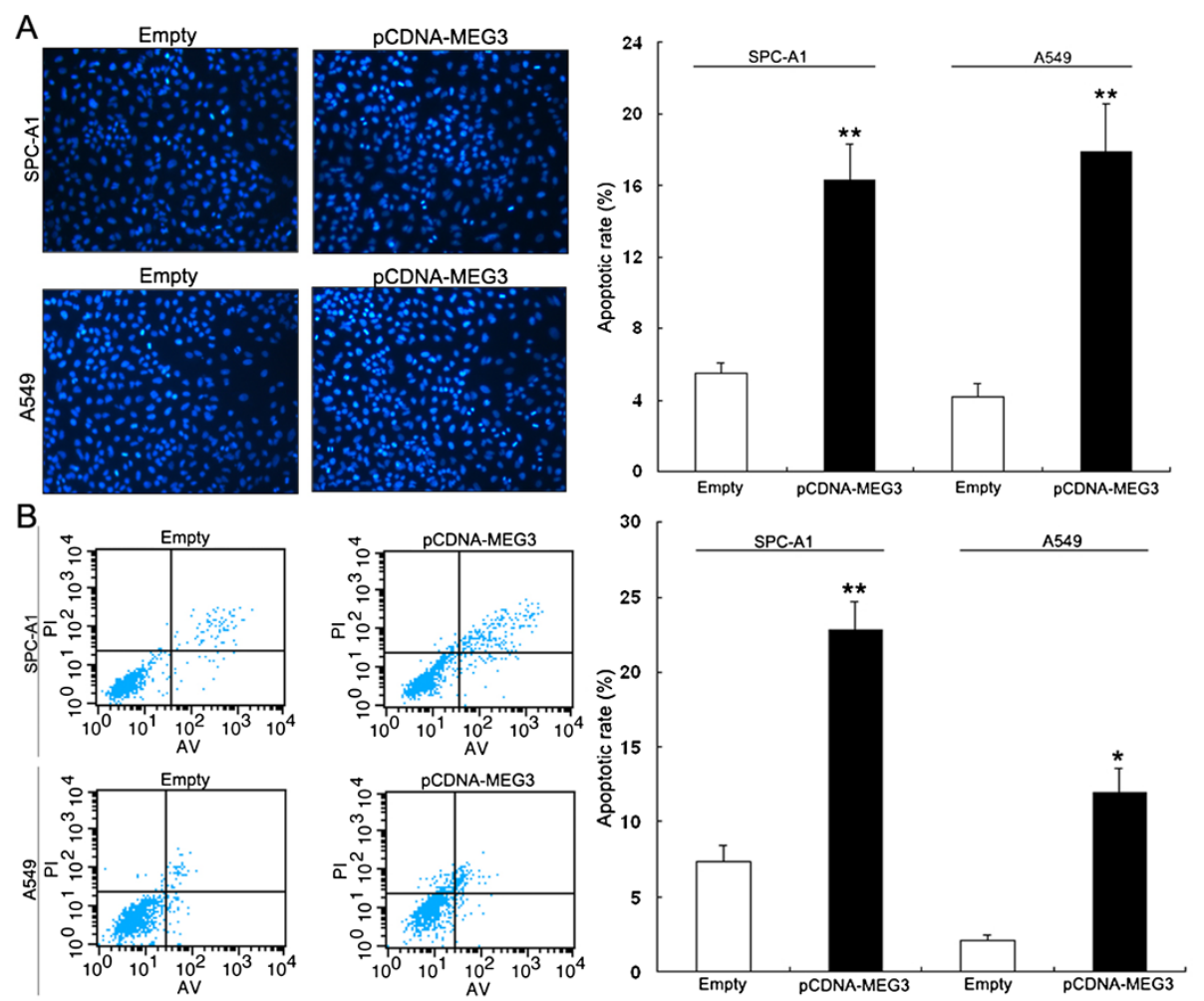

Figure 4 Effects of MEG3 on cell apoptosis. (A) Hoechst staining assay for cell apoptosis; the percentage of Hoechst-positive nuclei per optical field (at least 50 fields) was counted. (B) The apoptotic rates of cells were detected by flow cytometry. ${ }^{*} \mathrm{P}<0.05$ and ${ }^{*} \mathrm{P}<0.01$.

of lncRNAs may also affect epigenetic information and provide a cellular growth advantage, resulting in progressive and uncontrolled tumor growth $[10,16,33,34]$. Although IncRNAs may have impact on human cancers, the basis of their molecular mechanisms is still not well known. Therefore, the interplay between proteins and lncRNAs is an important topic in the field of cancer biology, in which lncRNAs may provide the missing clue of the well-known oncogenic and tumor suppressor network.

To date, many lncRNAs have been identified, and their involvement in human cancer has been extensively reported. The lncRNA MALAT-1 expression was markedly increased in primary bladder tumors that subsequently showed evidence of metastasis, and its overexpression could promote bladder cancer cells invasion by modulating epithelial-mesenchymal transition (EMT)-associated ZEB1, ZEB2, Slug and E-cadherin levels or by activating Wnt signaling [35]. In this study, we found that the expression of IncRNA MEG3 was decreased in NSCLC tissues when compared to normal tissues. Specifically, MEG3 expression was found to be significantly lower at later stages of tumor development and in tumors that had undergone increase in size. Moreover, the overall survival time of patients with moderate or strong MEG3 expression levels was significantly higher than that of patients with lower MEG3 expression levels. Moreover, loss or significant reduction of
MEG3 expression in various human primary tumors including neuroblastomas, hepatocellular cancers and gliomas has been well documented [21,36,37]. In addition, we demonstrate that MEG3 expression is lost in multiple NSCLC cell lines compared to a normal human bronchial epithelial cell line (16HBE). Similarly, loss of MEG3 expression has also been found in many cancer cell lines including those derived from brain, bladder, bone marrow, breast, cervix, colon, liver, lung, meninges and prostate [18]. We also showed that DNA methylation may underlie the lost expression of MEG3 in NSCLC tissues. This suggests that the decreased expression of MEG3 may be mediated by DNA methylation and useful in the development of novel prognostic or progression markers for NSCLC.

In order to highlight the impact of dysregulated expression and function of MEG3, we show the critical role of MEG3 in the development of NSCLC. Ectopic expression of MEG3 by transfection decreased the cell growth, and led to the promotion of cell apoptosis in vitro and in vivo. To further investigate how MEG3 induces NSLCC cells apoposis and growth arrest, we examined the level of p53 after transfection of pCDNA-MEG3 in SPCA1 cells. We found that re-expression of MEG3 could significantly stimulate the level of p53 protein. Peng-jun Wang and Yunli Zhou have also reported that non-coding RNA MEG3 may function as a tumor suppressor mediated 

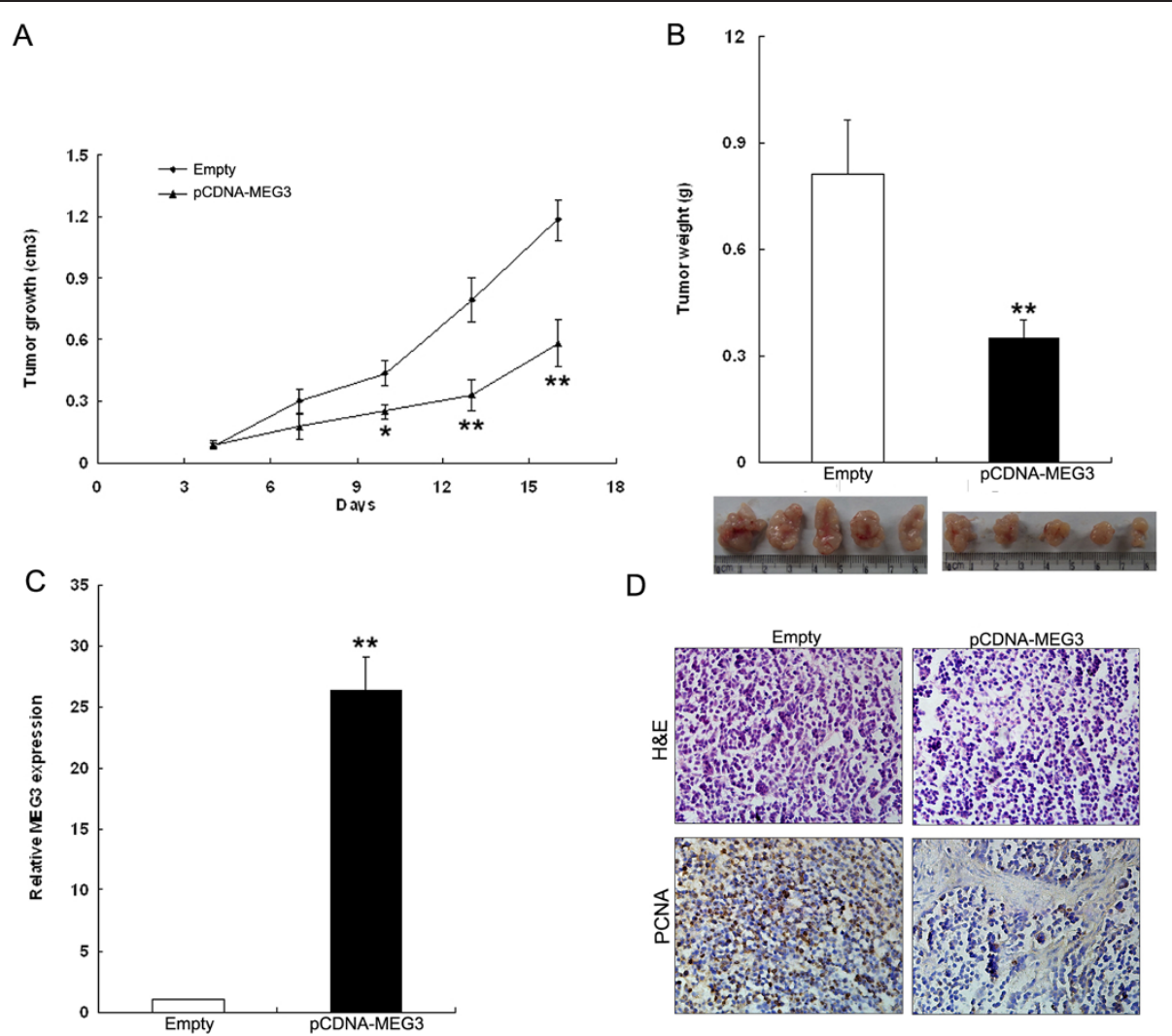

D

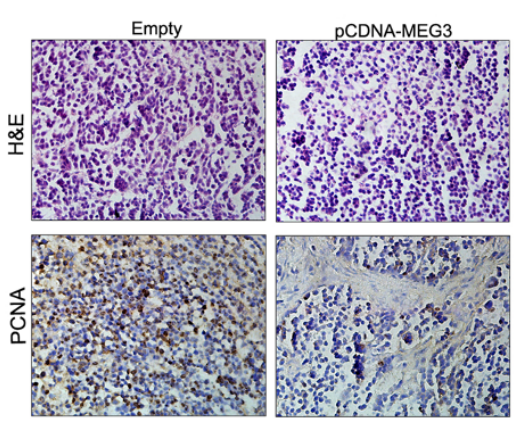

Figure 5 Effects of MEG3 on tumor growth in vivo. (A) The tumor volume was calculated once every three days after injection of SPC-A1 cells stably transfected with PCDNA-MEG3 or empty vector. Points, mean $(n=3)$; bars indicate S.D. (B) Tumor weights are represented as means of tumor weights \pm s.d. (C) QPCR analysis of MEG3 expression in tumor tissues formed from SPC-A1/MEG3, SPC-A1/NC. (D). Tumors developed from PCDNA-MEG3 transfected SPC-A1 cells showed lower PCNA protein levels than tumors developed by control cells. Upper: H \& E staining; Lower: immunostaining. ${ }^{*} P<0.05,{ }^{*} P<0.01$ and N.S. not significant.

by inducing the activation of p53 [21,22]. As an important transcription factor, $\mathrm{p} 53$ is capable of regulating expression of many target genes leading to the suppression of tumor development and growth, and it is mutated in most human cancers [38]. Generally, p53 level is very low due to rapid degradation via the ubiquitin-proteasome pathway. The ubiquitination of p53 is mainly mediated by MDM2, an E3 ubiquitin ligase. Inhibition of MDM2 plays a major role in p53 stabilization. A decrease in MDM2 protein level was observed in SPC-A1 cells transfected with PCDNA-MEG3,

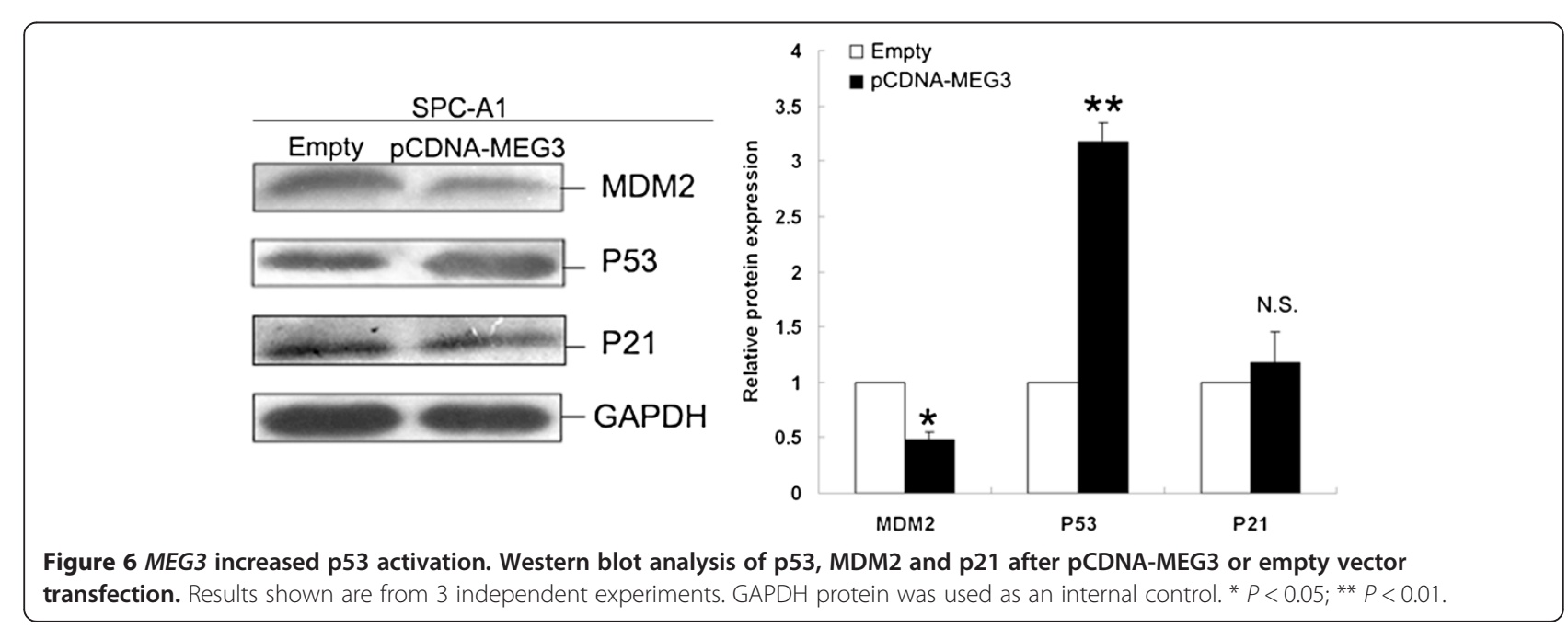


suggesting that MDM2 downregulation is one of the mechanisms by which MEG3 activates p53. Interestingly, the results revealed that $M E G 3$ does not stimulate $\mathrm{p} 21^{\mathrm{Cip} 1}$ expression, a well-known p53 target gene. These findings indicate that lncRNA MEG3 may function as a tumor suppressor by activating p53 and underlying target genes, but not $\mathrm{p} 21^{\mathrm{Cip} 1}$, and its deficiency or decreased expression or function could contribute to NSCLC development.

\section{Conclusions}

In summary, we demonstrate that the loss of lncRNA MEG3 expression is a common event underlying NSCLC, suggesting that MEG3 may play a key functional role in NSCLC developmeng and as a negative prognostic factor for NSCLC patients and an indicative of poor survival rates. The current study provides novel role of lncRNAs, specifically MEG3, and may help us to better understand the pathogenesis and development of NSCLC. Further understanding of this mechanism will foster the development of IncRNA-directed diagnostic and therapeutic agents against NSCLC.

\section{Additional files}

Additional file 1: Clinical data, such as age, gender, TNM stage at. Al of individual patients.

Additional file 2: Inhibition of MEG3 promotes cell proliferation in vitro. (A) Analysis of MEG3 expression levels in $\mathrm{H} 1299$ cells transfected with si-MEG3 or si-NC by qRTPCR. (B) MTT assay was performed to determine the proliferation of $\mathrm{H} 1299$ cells. Data represent the mean \pm S.D. from three independent experiments. (C) Colonyforming growth assays were performed to determine the proliferation of H1299 cells. The colonies were counted and captured.

\section{Competing interests}

The authors declare that they have no competing interests.

\section{Authors' contributions}

$L K H, L W$ and XWP were involved in the conception and design of the study. ZML and WWQ was involved in the provision of study material and patients. $\mathrm{LXH}, \mathrm{SM}$ and HYY performed the data analysis and interpretation. LKH wrote the manuscript. XWP approved the final version. All authors read and approved the final manuscript.

\section{Acknowledgments}

This work was supported by the Funds of the National Natural Science Foundation of China (WPXie) under Contract No. 8127357 and (HWang) under Contract No. 30971319 and Health Promotion Project of Jiangsu funded by the Priority Academic Program Development of Jiangsu Higher Education Institutions. We would like to acknowledge Professor Wei De for providing useful advice during the design of experiments.

This work was supported by the Funds of the National Natural Science Foundation of China (KHLu) under Contract No. 81372397.

\section{Author details}

'Department of Oncology, First Affiliated Hospital, Nanjing Medical University, Nanjing, People's Republic of China. ${ }^{2}$ Department of Biochemistry and Molecular Biology, Nanjing Medical University, Nanjing, People's Republic of China. ${ }^{3}$ Department of respiratory, First Affiliated Hospital, Nanjing Medical University, Nanjing, People's Republic of China.

${ }^{4}$ Immunology and Reproductive Biology Lab of Medical School and State
Key Laboratory of Pharmaceutical Biotechnology, Nanjing University, Nanjing, People's Republic of China.

Received: 11 April 2013 Accepted: 17 September 2013

Published: 7 October 2013

\section{References}

1. Jemal A, Siegel R, Xu J, Ward E: Cancer statistics, 2010. CA Cancer J Clin 2010, 60(5):277-300.

2. Verdecchia A, Francisci S, Brenner H, Gatta G, Micheli A, Mangone L, Kunkler I: Recent cancer survival in Europe: a 2000-02 period analysis of EUROCARE-4 data. Lancet Oncol 2007, 8(9):784-796.

3. Liu XH, Lu KH, Wang KM, Sun M, Zhang EB, Yang JS, Yin DD, Liu ZL, Zhou J, Liu ZJ, et al: MicroRNA-196a promotes non-small cell lung cancer cell proliferation and invasion through targeting HOXA5. BMC Cancer 2012, $12: 348$.

4. Gutschner T, Hammerle M, Eissmann M, Hsu J, Kim Y, Hung G, Revenko AS, Arun $G$, Stentrup M, Gross M, et al: The non-coding RNA MALAT1 is a critical regulator of the metastasis phenotype of lung cancer cells. Cancer Res 2013, 73(3):1180-1189.

5. Ponting $C P$, Oliver $P L$, Reik W: Evolution and functions of long noncoding RNAs. Cell 2009, 136(4):629-641.

6. Wilusz JE, Sunwoo H, Spector DL: Long noncoding RNAs: functional surprises from the RNA world. Genes Dev 2009, 23(13):1494-1504.

7. Gupta RA, Shah N, Wang KC, Kim J, Horlings HM, Wong DJ, Tsai MC, Hung T, Argani P, Rinn JL, et al: Long non-coding RNA HOTAIR reprograms chromatin state to promote cancer metastasis. Nature 2010, 464(7291):1071-1076.

8. Loewer S, Cabili MN, Guttman M, Loh YH, Thomas K, Park IH, Garber M, Curran $M$, Onder T, Agarwal $S$, et al: Large intergenic non-coding RNA-RoR modulates reprogramming of human induced pluripotent stem cells. Nat Genet 2010, 42(12):1113-1117.

9. Wang J, Liu X, Wu H, Ni P, Gu Z, Qiao Y, Chen N, Sun F, Fan Q: CREB upregulates long non-coding $R N A, H U L C$ expression through interaction with microRNA-372 in liver cancer. Nucleic Acids Res 2010, 38(16):5366-5383.

10. Khaitan D, Dinger ME, Mazar J, Crawford J, Smith MA, Mattick JS, Perera RJ: The melanoma-upregulated long noncoding RNA SPRY4-IT1 modulates apoptosis and invasion. Cancer Res 2011, 71(11):3852-3862.

11. Yang F, Zhang L, Huo XS, Yuan JH, Xu D, Yuan SX, Zhu N, Zhou WP, Yang GS, Wang $Y Z$, et al: Long noncoding RNA high expression in hepatocellular carcinoma facilitates tumor growth through enhancer of zeste homolog 2 in humans. Hepatology 2011, 54(5):1679-1689.

12. Cui Z, Ren S, Lu J, Wang F, Xu W, Sun Y, Wei M, Chen J, Gao X, Xu C, et al: The prostate cancer-up-regulated long noncoding RNA PIncRNA-1 modulates apoptosis and proliferation through reciprocal regulation of androgen receptor. Urol Oncol 2013, 31(7):1117-1123.

13. Wang Y, Chen W, Yang C, Wu W, Wu S, Qin X, Li X: Long non-coding RNA UCA1a(CUDR) promotes proliferation and tumorigenesis of bladder cancer. Int J Oncol 2012, 41(1):276-284.

14. Jin G, Sun J, Isaacs SD, Wiley KE, Kim ST, Chu LW, Zhang Z, Zhao H, Zheng SL, Isaacs WB, et al: Human polymorphisms at long non-coding RNAs (IncRNAs) and association with prostate cancer risk. Carcinogenesis 2011, 32(11):1655-1659.

15. Geng YJ, Xie SL, Li Q, Ma J, Wang GY: Large intervening non-coding RNA HOTAIR is associated with hepatocellular carcinoma progression. J Int Med Res 2011, 39(6):2119-2128.

16. Kim K, Jutooru I, Chadalapaka G, Johnson G, Frank J, Burghardt R, Kim S, Safe S: HOTAIR is a negative prognostic factor and exhibits prooncogenic activity in pancreatic cancer. Oncogene 2013, 32(13):1616-1625.

17. Kotake Y, Nakagawa T, Kitagawa K, Suzuki S, Liu N, Kitagawa M, Xiong Y: Long non-coding RNA ANRIL is required for the PRC2 recruitment to and silencing of p15(INK4B) tumor suppressor gene. Oncogene 2011, 30(16):1956-1962.

18. Zhou $Y$, Zhang $X$, Klibanski A: MEG3 noncoding RNA: a tumor suppressor. J Mol Endocrinol 2012, 48(3):R45-R53.

19. Benetatos L, Vartholomatos $G$, Hatzimichael E: MEG3 imprinted gene contribution in tumorigenesis. Int J Cancer 2011, 129(4):773-779.

20. Anwar SL, Krech T, Hasemeier B, Schipper E, Schweitzer N, Vogel A, Kreipe $\mathrm{H}$, Lehmann U: Loss of imprinting and allelic switching at the DLK1MEG3 locus in human hepatocellular carcinoma. PLoS One 2012, 7(11):e49462. 
21. Wang $P$, Ren Z, Sun P: Overexpression of the long non-coding RNA MEG3 impairs in vitro glioma cell proliferation. J Cell Biochem 2012, 113(6):1868-1874.

22. Zhou Y, Zhong Y, Wang Y, Zhang X, Batista DL, Gejman R, Ansell PJ, Zhao J, Weng C, Klibanski A: Activation of p53 by MEG3 non-coding RNA. J Biol Chem 2007, 282(34):24731-24742

23. Zhang SZ: Knockdown of c-Met by adenovirus-delivered small interfering RNA inhibits hepatocellular carcinoma growth in vitro and in vivo. Mol Cancer Ther 2005, 4(10):1577-1584.

24. Kilkenny C, Browne W, Cuthill IC, Emerson M, Altman DG: Animal research: reporting in vivo experiments: the ARRIVE guidelines. J Gene Med 2010, 12(7):561-563.

25. Derrien T, Johnson R, Bussotti G, Tanzer A, Djebali S, Tilgner H, Guernec G, Martin D, Merkel A, Knowles DG, et al: The GENCODE v7 catalog of human long noncoding RNAs: analysis of their gene structure, evolution, and expression. Genome Res 2012, 22(9):1775-1789.

26. Huarte M, Guttman M, Feldser D, Garber M, Koziol MJ, Kenzelmann-Broz D, Khalil AM, Zuk O, Amit I, Rabani M, et al: A large intergenic noncoding RNA induced by p53 mediates global gene repression in the p53 response. Cell 2010, 142(3):409-419.

27. Birney E, Stamatoyannopoulos JA, Dutta A, Guigo R, Gingeras TR, Margulies EH, Weng Z, Snyder M, Dermitzakis ET, Thurman RE, et al: Identification and analysis of functional elements in $1 \%$ of the human genome by the ENCODE pilot project. Nature 2007, 447(7146):799-816.

28. Louro R, Smirnova AS, Verjovski-Almeida S: Long intronic noncoding RNA transcription: expression noise or expression choice? Genomics 2009, 93(4):291-298.

29. Khalil AM, Guttman M, Huarte M, Garber M, Raj A, Rivea Morales D, Thomas K, Presser A, Bernstein BE, van Oudenaarden A, et al: Many human large intergenic noncoding RNAs associate with chromatin-modifying complexes and affect gene expression. Proc Natl Acad Sci U S A 2009, 106(28):11667-11672.

30. Spitale RC, Tsai MC, Chang HY: RNA templating the epigenome: long noncoding RNAs as molecular scaffolds. Epigenetics 2011, 6(5):539-543.

31. Wierzbicki AT: The role of long non-coding RNA in transcriptional gene silencing. Curr Opin Plant Biol 2012.

32. Huang Y, Liu N, Wang JP, Wang YQ, Yu XL, Wang ZB, Cheng XC, Zou Q: Regulatory long non-coding RNA and its functions. J Physiol Biochem 2012, 68(4):611-618.

33. Yuan SX, Yang F, Yang Y, Tao QF, Zhang J, Huang G, Wang RY, Yang S, Huo $X S$, Zhang L, et al: Long non-coding RNA-MVIH promotes angiogenesis and serves as a predictor for HCC patients' poor recurrence-free survival after hepatectomy. Hepatology 2012, 56(6):2231-2241.

34. Guttman M, Amit I, Garber M, French C, Lin MF, Feldser D, Huarte M, Zuk O, Carey BW, Cassady JP, et al: Chromatin signature reveals over a thousand highly conserved large non-coding RNAs in mammals. Nature 2009, 458(7235):223-227.

35. Ying L, Chen Q, Wang Y, Zhou Z, Huang Y, Qiu F: Upregulated MALAT-1 contributes to bladder cancer cell migration by inducing epithelial-to -mesenchymal transition. Mol Biosyst 2012, 8(9):2289-2294.

36. Braconi C, Kogure T, Valeri N, Huang N, Nuovo G, Costinean S, Negrini M, Miotto E, Croce CM, Patel T: microRNA-29 can regulate expression of the long non-coding RNA gene MEG3 in hepatocellular cancer. Oncogene 2011, 30(47):4750-4756.

37. Astuti D, Latif F, Wagner K, Gentle D, Cooper WN, Catchpoole D, Grundy R, Ferguson-Smith AC, Maher ER: Epigenetic alteration at the DLK1-GTL2 imprinted domain in human neoplasia: analysis of neuroblastoma, phaeochromocytoma and Wilms' tumour. Br J Cancer 2005, 92(8):1574-1580.

38. Vousden $\mathrm{KH}$, Prives $\mathrm{C}$ : Blinded by the Light: the growing complexity of p53. Cell 2009, 137(3):413-431.

doi:10.1186/1471-2407-13-461

Cite this article as: Lu et al:: Long non-coding RNA MEG3 inhibits NSCLC cells proliferation and induces apoptosis by affecting p53 expression. BMC Cancer 2013 13:461.

\section{Submit your next manuscript to BioMed Central and take full advantage of:}

- Convenient online submission

- Thorough peer review

- No space constraints or color figure charges

- Immediate publication on acceptance

- Inclusion in PubMed, CAS, Scopus and Google Scholar

- Research which is freely available for redistribution

Submit your manuscript at www.biomedcentral.com/submit
C) Biomed Central 